\title{
Promoting Intelligent Energy Use Needs Collaboration
}

The Europhysics Study Conference Using Energy in an Intelligent Way (Trassenheide, 6-10 May 1993) organized in collaboration with the WE-Heraeus Foundation identified the gap between scientists on the one hand and economists and politicians on the other as the main problem in defining research goals. It has led to energy being considered as a resource and not a production factor, so only $4 \%$ of a developed country's GNP is accounted for by energy, while a massive $75 \%$ covers labour. However, an overall energy policy within which research objectives can be structured will not emerge from short-term political expediency based on advice from specialists who only talk to colleagues.

Physicists must therefore teach, and learn from, others. The task may not be so difficult: economists, for instance, understand mathematical optimization and behavioural scientists and psychologists can show us how to convince people of the need for rational energy policies. B. Gonsior (Ruhr University, Bochum, and Chairman of the meeting) in his introduction argued that one will have to tackle energy issues by "pulling together the pieces within their social context". So the EPS Action Committee for Physics and Society (ACPS) which helped organize the event is an appropriate forum. K. Rebane (Institute of Physics, Tartu) in his summary felt developing strategies will not be easy as evolution and man's history indicate that the "winners have been those species and societies who act quickly, consume high-quality energy and materials, and pay little or no attention to long-term consequences"

Knowing what to say will not be enough as reasoning must also be communicated to the public at large. A conclusion of a discussion session convened by the 30 participants was that one should exploit the fact that decision makers who define boundaries and rules for intelligent action are sensitive to the media. The fundamental message - that we must address the unavoidable production of entropy and not energy conservation (which is automatically guaranteed by the laws of physics) - and its corollary - that economic calculations should be based more on entropy than on energy - have not yet taken root. One strategy may be to formulate positions which can be defended by the physics community. However, there were no specific decisions on priorities for future action; these should emerge following an analysis by the ACPS and a discussion of the report* of the meeting during the symposium Physics in a Changing World at the EPS-9 General Conference (Florence, 14-17 September 1993).

\section{Clear Objectives}

One of the meeting's aims was to review progress following the American Physical Society's (APS) landmark study in 1975 to identify areas of research which held promise of improved technology by bringing a physics approach to bear on energy issues. The conclusion was that fundamental understanding had not advanced greatly. Physicists, however, have made useful contributions to the formulation of an intelligent research agenda.
It was unfortunate that owing to last-minute difficulties, R.H. Socolow, who led the 1975 APS study and is planning a new one, could not attend. R. Dekeyser, the ACPS Secretary, reviewed instead three recent papers by Socolow, who is optimistic that growth and full development in the third world are compatible with environmental objectives (see below). Energy issues will be come deeply embedded in human attitudes, implying an abundance of new technology, especially for developing countries where there must be "technological leap-frogging" and not simply transplantation.

The argument that well-designed government policies will be good for the economy was taken up by several participants. G. De Lepeleire, an engineer from the University of Leuven who advises the Belgian government on energy issues, proposed transferring taxes from employment to energy to give a dramatic decrease in the ratio of labour to energy costs. This would stimulate both energy saving and employment - a strategy which is appropriate in Europe where social charges are high. R. Kümmel from the University of Würzburg and an advisor to Germany's energy minister, felt that measures must be taken to decrease energy consumption for "unreasonable" purposes by taxing fossil fuels to make non-fossil energy sources economically competitive, the extra revenue being used to inform the public and to boost the development of non-fossil options. The usual justification for such a strategy is the high real cost of fossil fuels owing to entropy production involving both thermal and chemical emissions.

The emission situation was reviewed by B. Gonsior: "business as usual" models estimate worldwide energy consumption in 2020 of 11.2 to $17.2 \mathrm{TWa} / \mathrm{a}$, with industrial countries consuming 3.2-8.5 kW per capita $(p c)$ and the third world only 1.1-1.4 kW pc. Consumption in 1990 was $10 \mathrm{TWa} / \mathrm{a}$ or $2 \mathrm{~kW} \mathrm{pc}$ so the production of greenhouses gases would double. For $4 \mathrm{~kW} \mathrm{pc}$ (the lower limit) and a doubling of population in 50 years, we have a fourfold increase in energy demand that will be difficult to meet. He advocated $2 \mathrm{~kW} p c$ as a target, close to the $1 \mathrm{GAG}$ (greatest acceptable grant) of $1.5 \mathrm{~kW} \mathrm{pc}$ (or $36 \mathrm{kWh} /$ day $p c$ ) proposed in 1981. It is com- patible with projections that consumption in industrialised countries can be halved by 2020 , and that a western standard of living could be achieved in the third world with a $30 \%$ increase in their consumption. Conscious that some countries consumed close to $10 \mathrm{~kW} p c$ in 1983, he invited participants to envisage "what a sustainable world would look like" by drawing up their own menus of energy consumption, remembering that $20000 \mathrm{~km}$ by car needs $24 \mathrm{kWh}$. Research, meanwhile, should focus second-law thermodynamics on specific energy-using tasks to define minimum energy paths and standards of efficiency for energy conversion.

\section{Fundamentals Examined}

Before addressing systems and technology designed in the physics perspective of second-law efficiencies, A.S. Silbergleit (A.F. loffe Physicotechnical Institute, St. Petersburg) gave a detailed analysis of the laws of thermodynamics. The second law assumes an integrating factor (the temperature) which is the same for all thermodynamic systems. This universality can be checked by seeking a single-parameter system whose integrating divisor (temperature) is not the absolute gas temperature. He claimed a rotating wheel with spokes made from a shape-memory metal may be such a system. More significantly, ideal processes in energy conversion systems based on cyclic changes of the thermodynamic properties of a working body have zero power, while actual devices involve irreversible energy losses due to heat flow. One may be able to escape these constraints on energy conversion by exploiting a strongly non-equilibrium open system that has a device using a working fluid with a high degree of self-organization (giving negative entropy production). One possibility is a socalled vortex turbine based on the condensation of a vapour vortex on the inside of a rotating cone. While the concept remains to be verified experimentally (and this will be difficult), it at least highlights the importance of analysing the efficiencies of irreversible processes.

Designing specific energy conversion systems and processes will, in general, require numerical simulation in addition to exact analysis owing to their complexity. S. Wirz (Ruhr University, Bochum) described, for example, a sophisticated, computer-based modelling tool for combustors. Industrial interest in this type of approach has waned considerably in the present era of cheap energy so it is perhaps not surprising that his was the only

Participants at the IIIth WE-Heraeus Seminar Using Energy in an Intelligent Way organized by the EPS Action Committee for Physics and Society.

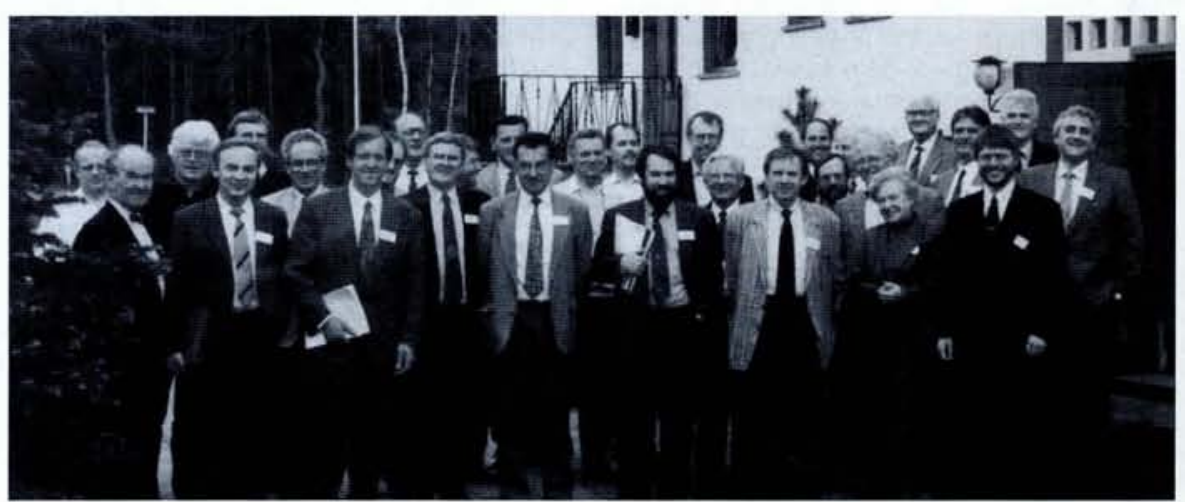


presentation in this important area. The situation may also account for comments by other German groups that development and engineering skills have been "wasted" owing to the absence of a long-term commitment to energy conservation.

\section{Sophisticated Systems Analysis}

J. Gretz (JRC, Ispra) who manages the European Community's (EC) hydrogen in transport programme on behalf of the EC Parliament (hydrolytically produced $\mathrm{H}_{2}$ is shipped from Canada for a fleet of buses which are are already operating and for a $\mathrm{H}_{2}$-powered passenger aircraft due to fly next year) analysed the limiting performance of specific systems. Solar energy conversion is limited to about $70 \%$ efficiency, while the global efficiency of photosynthesis, in spite of a $33 \%$ quantum efficiency, is below $1 \%$; the influence on the climate of a solar power plant is proportional to the difference between its conversion efficiency and that of the terrain it replaces (currently fairly small, but likely to increase); electrolysis is more efficient overall than thermochemical cycles for producing $\mathrm{H}_{2}$ (belief in the opposite misdirected research for many years - a potent reminder of the importance of careful analy sis). Atmospheric $\mathrm{CO}_{2}$ can be managed using forestry (foresting 6\% of the Earth's surface will absorb all of the $\mathrm{CO}_{2}$ produced today) so it was fitting that J.T. McMullan, who heads the the University of Ulster's Centre for Energy Research, described a detailed analysis of power generation from wood combustion. A $1000 \mathrm{t} /$ day plant is optimum, and the UK has enough productive woodland to support 60 plants generating
$3000 \mathrm{MW}$ in a neutral way with respect to greenhouse gas emissions.

According to W. Eichhammer and $\mathrm{E}$. Jochem (Fraunhofer Institute, Karlsruhe) the potential for energy (and entropy) savings in large-scale, regional energy supply systems will be more important in the domestic sector than in industry. Experience with gas-driven heat pumps for air-conditioning in Japan and with co-generation units in Denmark has shown the value of consistent, long-term planning in the domestic sector. Potential savings in transport are also large, and R.D. Kühne (Steierwald Schönharting $\mathrm{GmbH}$, Stuttgart) described how government policies are being used to reverse the trend towards modes involving high energy consumption.

C.D. Andriesse (University of Utrecht) took the case of co-generation to demonstrate the utility of fairly simple analyses for "braking entropy production". A device producing slightly more kinetic energy than heat is optimum, and given the relative cost of transporting electricity and heat, it should be rated at about $10 \mathrm{MW}$. A more sophisticated stochastic optimization model described by $R$. Kümmel predicted roughly $20 \%$ reductions in $\mathrm{CO}_{2}$ production and energy consumption for a German city using local co-generation. But the $40-50 \%$ increase in costs are only economic in the entrepreneurial sense if energy prices double, or even triple. Extending models requires much more detailed information on energy demand along the lines being addressed by a new 50 MDM German project called IKARUS, probably the largest study of its type in Europe.

Although technology will be a necessary but not a sufficient condition for energy con- servation, this is not the reason why contributions on energy conversion and saving technologies and materials will not be summarised. It is simply too difficult to do justice to the many physics concepts involved in the topics presented (convective thermal rectification, thermoionics, photovoltaics, combustion diagnosis, light concentration, spectrally selective materials). Readers are referred instead to the report of the meeting. Little was said about monitoring and datataking, aspects of entropy braking in the widest sense to which physics increasingly contributes.

- Using Energy in an Intelligent Way, Proc. 111th WE-Heraeus Seminar, 6-10 May 1993, Trassenheide, Germany; Ed.: E.W.A. Lingeman (to published by EPS; price: SFR 70-).

P.G. Boswell

\section{EPS High-Energy and Particle Physics Prize}

The High-Energy and Particle Physics Prize of EPS, awarded every two years through the High-Energy and Particle Physics Division, has been won this year by Martinus J.G. ("Tini") Veltman of The University of Michigan, Ann Arbor, USA, for pioneering work on the rôle of massive Yang-Mills theories for weak interactions. The prize will presented at the International Europhysics High-Energy Physics Conference, Marseilles, 22-28 July 1993. Previous winners are G. Charpak (1989) and N. Cabibbo (1991).

\section{Why MathSoft Overseas has a centre of excellence for Mathematica at CERN}

\section{Because Mathematica responds to CERN's needs for a comprehensively supported mathematical software package for physics and engineering.}

Mathematica represents a genuine revolution in the art of computing.

Collects in a single package available on any computer:

- Symbolics: Integrate [Exp[- $\left.\left.\mathrm{x}^{\wedge} 2 / 2\right], \mathrm{x}\right] \rightarrow \mathrm{Sqrt}[\mathrm{Pi} / 2] \operatorname{Erf}[\mathrm{x} / \mathrm{Sqrt}[2]]$

- Numerics: Erf[1.2] $\rightarrow 0.910314$

- Graphics: Plot[Erf[x],\{x,-4,4\}, AxesLabel $\rightarrow$ ["x","y"\}]

- List processing: Erf[\{1.1,2.5,-3.4\}] $\rightarrow\{0.7747609,0.9991862,-0.9999965\}$

- Functional programming with pattern recognition:

Factorial function $\rightarrow$ fac[n_Integer/;n>0]: $=\mathrm{n}$ fac $[\mathrm{n}-1] ;$ fac $[0]=1$

- The Descartes ${ }^{\mathrm{TM}}$ Library extends Mathematica

- Euclidian geometry: all Euclidian geometry on point, straight, circle, conics, lines, and polygons. Application to rigorous drawing, theorem testing, CAD, desktop publishing, and any graphics technique.

- Differential geometry of curves and surfaces.

- Visualization: pre-processing of objects to be visualized in the form of Cartesian surfaces, parametrric lines and surfaces, and geometric solids; data representation in needle tower or interpolating surfaces. Interface to AVS ${ }^{\mathrm{TM}}$.

- Integration of Mathematica by MathSoft Overseas in process control using UNIX

- Dedicated technical and commercial support

- Optimum pricing (volume discount, educational grants, site licensing, etc.).

- Three-day courses: Introduction to Mathematica, symbolic programming.

- Consulting on specific problems.

To become a centre of excellence in Mathematica, please contact:

\section{MathSoft Overseas, CP 641, CH-1211 Geneva 3}

Tel.: +41-22-346 52 60; Fax: +41-22-346 5939

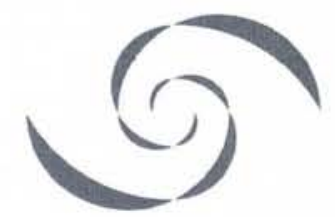

Visualization of a parametric surface

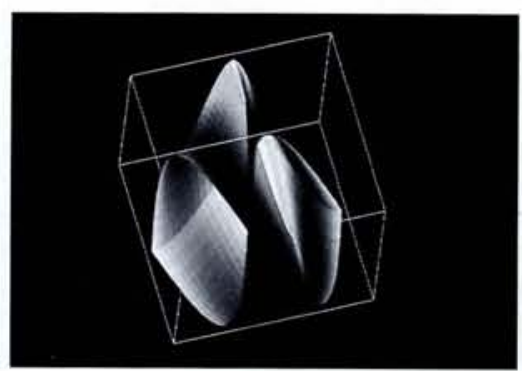

A figure produced with the statement: View $\left[\left\{\operatorname{Cos}[u], \operatorname{Sin}[v], u^{*} v / 10\right\},\{u,-P i, P i\},\{v,-P i, P i]\right]$. Descartes Visualization acts as the link between Mathematica and the AVSTM visualization program. The rigorously defined object could, for example, represent stream flow. 